\title{
A Fibre Bragg Grating Interrogation Technique Based on High Birefringence Fibre Loop Mirror and WDM
}

\author{
Xun Zhang ${ }^{1}$ Shuyang $\mathrm{Hu}^{1, \mathrm{a}}$ and Chuan $\mathrm{He}^{1}$ \\ ${ }^{1}$ School of applied Sciences, Beijing University of Technology, Beijing, 100124, China
}

\begin{abstract}
In this paper, a fibre Bragg grating (FBG) interrogation technique based on high Birefringence fibre loop mirror (Hi-Bi FLM) and wavelength division multiplexer (WDM) is proposed and demonstrated. The approximate linear edge of the transmittance of the Hi-Bi FLM, which is a sinusoidal function of wavelength, is used to interrogate the sensing FBGs and WDMs is used to realize wavelength discrimination .Suitable for both static and dynamic sensing, this interrogation method has the advantages of all fibre design and high stability.
\end{abstract}

\section{Introduction}

Fibre Bragg grating has been applied broader and broader because of its many advantages. The critical aspect related to the practical use of an FBG sensor is the necessity of performing accurate measurement of the small wavelength shift associated with thermal and strain state changes. To facilitate the broad use of this class of sensors, compact, rugged, wide range and low cost interrogation systems are required. In addition, improvements in the fabrication of FBGs using phase mask techniques have reduced the cost of grating fabrication so that the interrogation unit, rather than the sensor, accounts for a large proportion of the cost of a complete sensing system. Among the often used technic such as Matched-filter interrogation technique [1] , interferometric Fourier transform technique [2] and the technic using tunable light source[3], the edge filter demodulation technic in which the FBG is interrogated by measuring the power of the output light has the advantages such as high demodulation speed, cheapness, convenience and both suitable for both static and dynamic measurement. On the other hand the optical fiber-loop mirror has been used in several applications, namely in optical communications like a terahertz optical asymmetric demultiplexer [4] or a nonlinear optical loop mirror [5]. In the optical sensor, it has been used as a Sagnac interferometer for temperature measurement [6]. High birefringence fiber loop mirror (Hi-Bi FLM) has been used as edge filter to interrogate the FBG sensors in recent years [7,8]. In 2001, Seunghwan Chung reported a FBG demodulating technic [9] using Hi-Bi FLM with the resolution of $2.12 \mu \varepsilon$. Zhouguang reported an edge filter demodulation method [10] using Hi-Bi FLM and demodulation range of $6 \mathrm{~nm}$ was reached. Although this technic has many advantages such as fast demodulation speed, it can only interrogate one FBG at the same time, which limits its wider application. In this paper, an interrogation technique based on Hi-Bi FLM and WGM which can interrogate multiple FBG sensors is studied.

\section{Operational principle}

\subsection{High-birefringnece fiber loop mirror}

High-birefringence fiber loop mirror is composed of a section of high-birefringence fiber, a polarization controller and a $3 \mathrm{~dB}$ coupler, as shown in Figure1. The incident beam splits into two beams through the $3 \mathrm{~dB}$ coupler. Then two beams interfere at the output port. The transmittance function is[11] :

High-birefringence Fiber

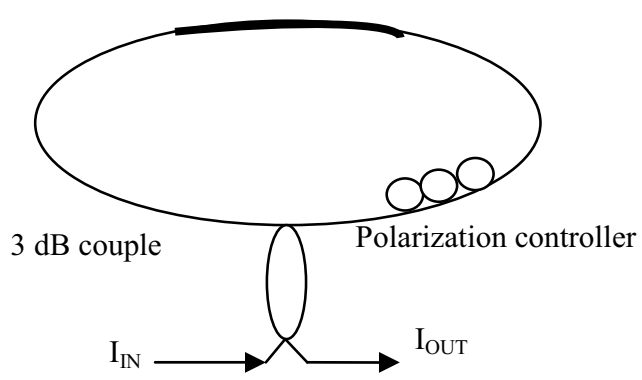

Figure1 Schematic diagram of high-birefringence fiber loop mirror

$$
\mathbf{T}=\left[\cos \frac{\pi 2 \bar{E}}{2} \sin \theta\right]^{2}
$$

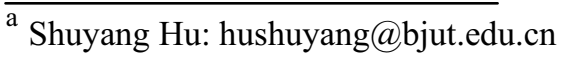


where $\mathrm{L}$ is the length of $\mathrm{HB}$ fiber, $\lambda$ is wavelength, $\mathrm{B}=\Delta \mathrm{n}=\left|n_{e}-n_{0}\right|$, is the difference of the index along fast and slow axes, $\theta$ (is a certain constant when Hi-Bi FLM having been installed )is the angle change of the polarization of the light propagating through the fiber

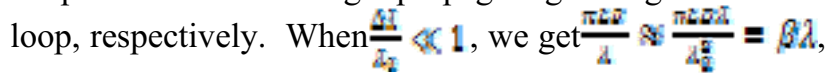
where $\beta=\frac{\pi L S}{A_{i}^{2}}, \Delta \lambda$ and $\lambda_{0}$ is the wavelength range and center wavelength of the source, respectively. We simplify (1) as:

$$
T=\frac{1+\cos \theta}{2} \sin ^{2} \theta
$$

From (2), we know that the transmission of HiBi-FLM is a sine function of $\lambda$ with the period of $\lambda^{2} / g_{L B}$, which is decided by $\mathrm{L}$ and $\mathrm{B}$. The transmission of the $\mathrm{f}$ HiBi-FLM we fabricated is shown in Figure 2, in which the curve A, $\mathrm{B}$ and $\mathrm{c}$ is the spectrum of light source, the light transmitting through the FLM and the transmittance, respectively.

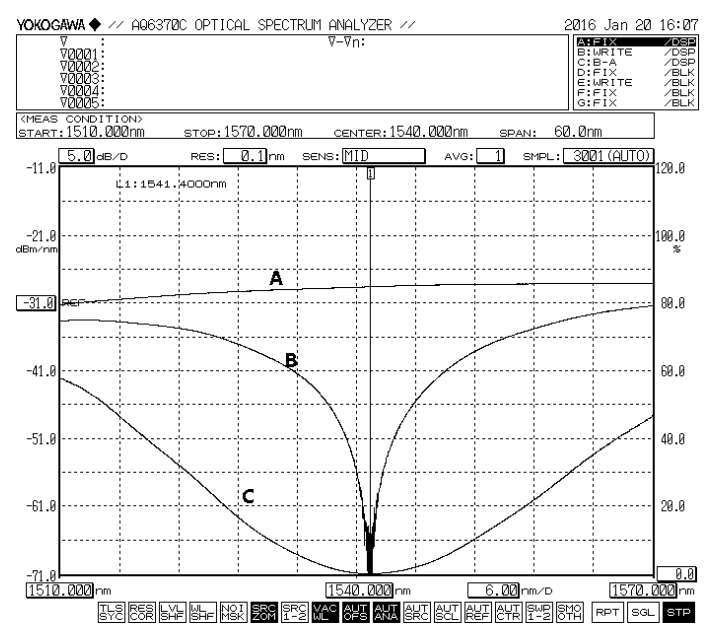

Figure2 The transmission spectrum of the Hi-Bi FLM

\subsection{Interrogation principle}

When the narrow band light reflecting by the FBG whose spectrum band width is narrow comparing with the period of transmission and its spectrum can be treated as Sfunction is launched into HiBi- FLM, the intensity of the light transmitted through is given by

$$
I_{t}=I_{l} T\left(\lambda_{-}\right)=\tau_{l} \frac{1+\cos \theta \pi \pi^{2}}{2} \sin ^{2} \theta
$$

Where $I_{1}$ and $\lambda_{a}$ is the intensity and wavelength of reflected light of FBG, respectively. So, the transmittance of the light reflectting from the sensing FBG and passing through the Hi-Bi FLM can be given as

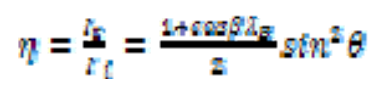

From (4) we know that we can interrogate the FBG by measuringๆ. Especially when the transmittance is in the nearly linear range the Hi-Bi FLM can be treated as a linear filter.
The Hi-Bi FLM and WDM based FBG interrogation system is carried out with the setup shown in Figure 3.The wide band light from SLED passes through coupler1 and enter the sensing FBGs. The reflecting light goes through coupler1 and is split into two beams via coupler 2. One of the beam goes through Hi-Bi FLM and WDM successively while the other beam only goes through the other WDM the same as the first. Eventually the light reflecting from different sensing FBGs is separated into different optical detector. Via measuring the corresponding beam going through FLM or not, the transmittance $(\eta)$ of FBGs can be calculated out and $\lambda_{B}$, the wavelength of FBGs can be determined.

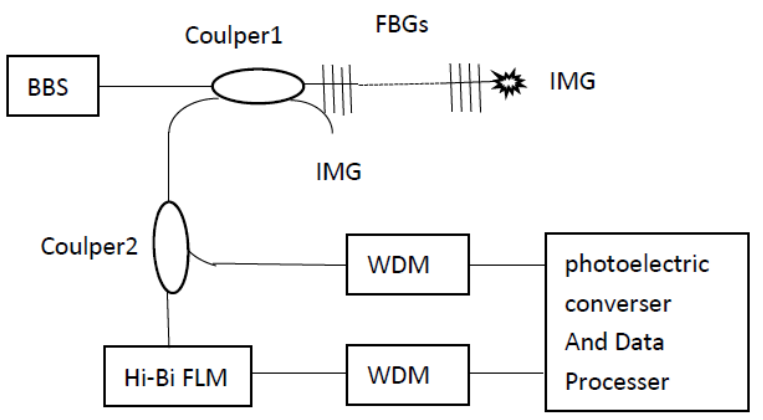

Figure 3 Schematic of the interrogation system

\section{Experimental Results}

In the experiment, CWDM is used to demonstrate the technique whose transmission spectrum is shown in Figure 4,in which $\mathrm{P} 1$ and $\mathrm{P} 2$ is the transmission spectrum of port1 and port3,respectively.SLED is used as light source and Hi-Bi FLM is made and the spectrum of them are both shown in Figure.1. Two FBGs with the center wavelength of $1520 \mathrm{~nm}$ and $1550 \mathrm{~nm}$ are used and the spectrum of their reflecting light are shown in Figure 5.

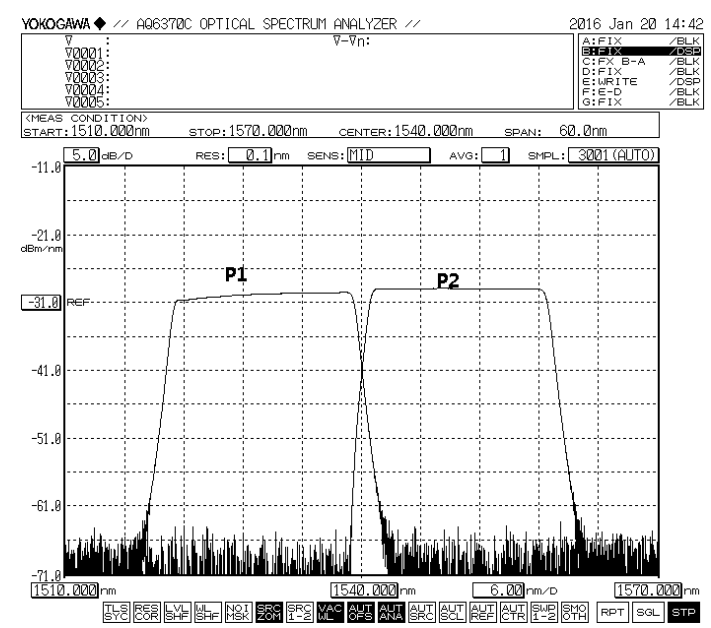

Figure 4 The spectrum of light passing through the WDM 


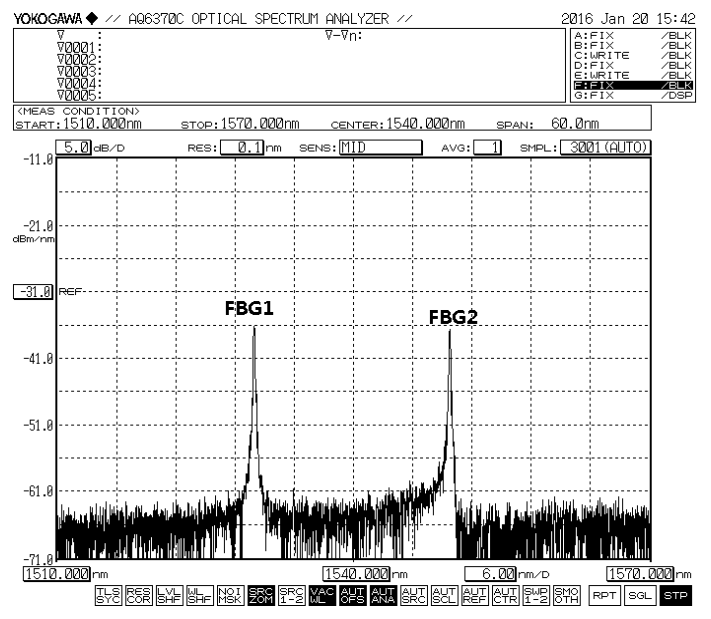

Figure 5 The reflective spectrum of the FBGs

The reflecting light of FBGs go through Hi-Bi FLM and WDM are separated into different detector and the two spectrum of FBG1 and FBG2 are shown in one picture in Figure 6. The wavelength of the FBGs can be interrogated by the value of $\eta$ which can be calculated by comparing the corresponding power of the light passing through Hi-Bi FLM or not.

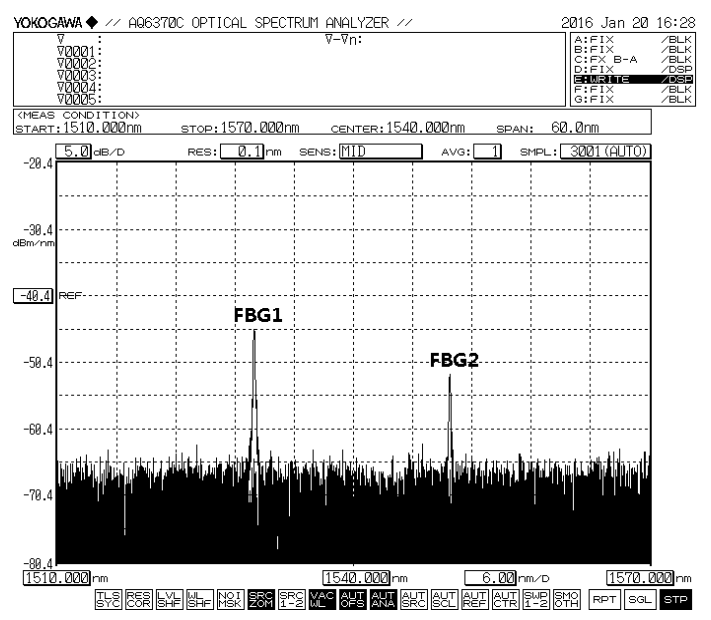

Figure.6 The reflective spectrum of the FBGs passing through WDM and Hi-Bi FLM

We adhere FBG1 to the equal deform beam to tune it which is shown in Figure 7. The beam is placed horizontal with one end is fixed while the other is free. So, we can tuning the wavelength of FBG by loading weight to the free end.

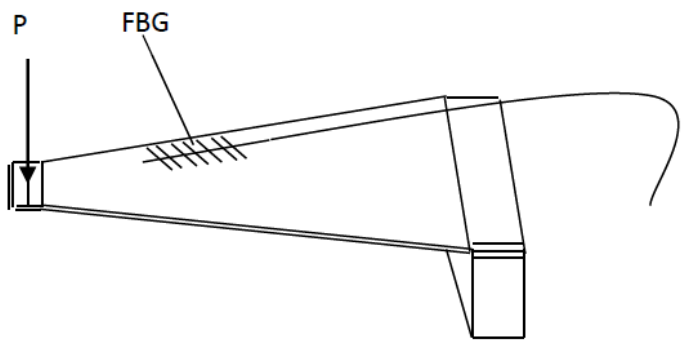

Figure 7 The schematic of wavelength tuning of FBG using equal deform beam.

By tuning the weight the transmission ( $\eta$ )varies with wavelength shift and the relationship between them is shown in Fig. 8, with $\mathrm{R}^{2}=0.9938$.

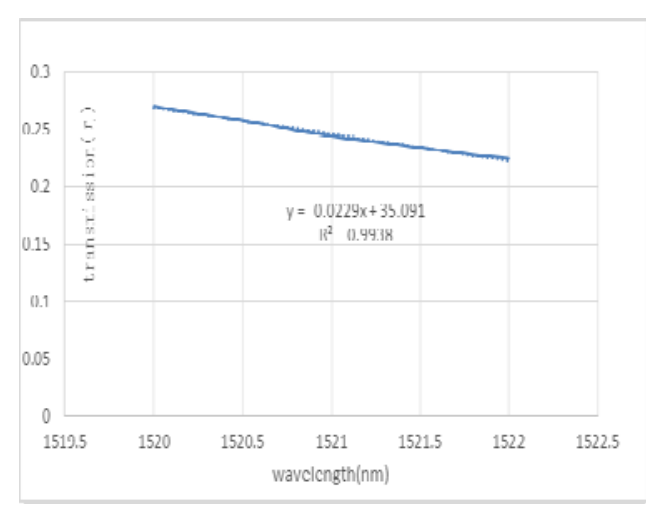

Figgure 8 Relationship between transmission and wavelength of FBG1

\section{Conclusions}

In this paper, a FBG interrogation technique based on HiBi FLM and WDM is proposed and demonstrated. With the capability of measure multiple FBG signal at the same time based on WDM, the technique has the advantages of fast demodulation, simple structure and suitable for both static and dynamic measuring.

\section{References}

1. Davis M A , Kersey A D. Matched-filter interrogation technique for fiber Bragg grating arrays Electro. Lett, 1995, 31(10):822 825

2. Ohn M M, Huang S Y, Measures R M, et al.. Arbitrary strain profile measurement within fiber gratings using interferometric Fourier transform technique Electron. Lett, 1997, 33(14):1242 1243

3. Br ady G, Kalli K, Webb D J, et al, Simultaneous interrogation of interferometric and Bragg grating sensors Opt .Lett ., 1995, 20(11):1340 1342 
4. J.P. Sokoloff, P.R. Prucnal, I. Glesk, and M. Kane, A terahertz optical asymmetric demux (TOAD), IEEE Photon Tech Lett 5 (1993), 787 - 790.

5. N.J. Doran and D. Wood, Nonlinear-optical loop mirror, Opt Lett 13 (1988), 56 - 58.

6. A.N. Starodumov, L.A. Zenteno, D. Monzon, and E. De La Rosa, Fiber Sagnac interferometer temperature sensor, Appl Phys Lett 70 (1997),19 21.

7. MORTIMORE D B, Fiber loop reflectors J. Lightwave Technol., 1988, 6(7):1217-1224.

8. Zhou Guang, Zhao Qida, Liu Yange, et al., Filtering Characteristics of fiber loop mirror filter composed of multi-stage high birefringent fibers Acta Optica Sinica 2004, 24(3):341-345.
9. SEUNGHWAN CHUNG, JUNGHO KIM, BONGAHN YU, et al., A Fiber Bragg Grating Sensor Demodulation Technique Using a Polarization Maintaining Fiber Loop Mirror IEEE Photonics Technology Letters, 2001, 3(2):1343-1346.

10. Zhou Guang, Zhao Qida,Kai Guiyun, et al., A novel edge filter demodulation method using high birefringence fiber loop mirror, Journal of Optoelectronics Laser, 2003, 14(12):1245-1249.

11. Yange Liu, Bo Liu, Xinhuan Feng, et al, Highbirefringence fiber loop mirrors and their applications as sensors, Applied Optics, 44(12): $2382-2390(2005)\}$ 